\title{
Reply: Breastfeeding-related maternal microchimerism
}

\author{
Jeremy M. Kinder, Ina A. Stelzer, Petra C. Arck and Sing Sing Way
}

We recently reviewed pregnancy-imprinted immunological shifts in mothers and offspring from the perspective of genetically foreign cells that establish microchimerism in both individuals after parturition (Immunological implications of pregnancy-induced microchimerism. Nat. Rev. Immunol. 17, 483-494 $(2017))^{1}$. Expanded tolerance between mothers and their offspring is widely conserved across mammalian species ${ }^{2}$, suggesting that the bidirectional transfer and long-term persistence of microchimeric cells are purposeful, with beneficial properties that outweigh any potential harmful immunological consequences ${ }^{3}$. Immunological acceptance of non-inherited maternal antigens (NIMAs) in offspring reinforces tolerance to matched antigens expressed by the developing fetus during next-generation pregnancies ${ }^{4,5}$. However, these cross-generational benefits require the overlap of antigenic traits between maternal grandmothers and their fetal grandchildren and can be diluted with increased polymorphism among individuals within a population. A more universal benefit of microchimeric maternal cells could involve their multilineage potential in replacing malfunctioning cells in a variety of infant and childhood autoimmune and autoinflammatory disorders ${ }^{6-9}$. In addition, a recent study reported detectable microchimeric maternal cells in the cord blood of human infants that decreased the risk of symptomatic malaria infection, but which were associated with increased parasitaemia risk ${ }^{10}$. Thus, regardless of commonality in polymorphic antigenic traits, microchimeric maternal cells likely instil in offspring important protective benefits with regard to optimal regeneration of vital tissues and dampened pathological inflammatory responses to microbial invaders.

In their Correspondence (Breastfeedingrelated maternal microchimerism. Nat. Rev. Immunol. http://dx.doi.org/10.1038/ nri .2017.115(2017) $)^{11}$, Moles and colleagues highlight the importance of maternal cells in breast milk and breast feeding for maintaining NIMA-specific tolerance. Along with the aforementioned cross-generational reproductive benefits ${ }^{4,5}$, another classic example of NIMA-specific tolerance is the improved long-term survival of human donor allograft tissue if it is matched for the recipient's non-inherited maternal HLA haplotypes ${ }^{12}$. Interestingly, the improved survival of NIMA-matched tissue allografts is overturned among individuals that were not breast fed ${ }^{13}$. A similar requirement for postnatal ingestion of maternal antigen occurs in animal crossfostering studies, in which elimination of the offspring's exposure to maternal breast milk overrides tolerance to NIMA-expressing donor allograft tissue ${ }^{14}$ and reduces the accumulation of immunosuppressive forkhead box protein $\mathrm{P} 3$-positive $\left(\mathrm{FOXP}^{+}\right)$regulatory $\mathrm{CD}^{+} \mathrm{T}$ cells $\left(\mathrm{T}_{\text {reg }}\right.$ cells) with NIMA specificity ${ }^{4,15}$. Importantly, however, postnatal ingestion of maternal antigens through breastfeeding alone does not confer immunological tolerance as cross-fostered mice neither accept NIMA-matched allografts nor have expanded levels of NIMA-specific FOXP3 ${ }^{+} \mathrm{T}_{\text {reg }}$ cells ${ }^{4,14}$. Thus, breastfeeding functionally potentiates, but does not bypass, the necessity for prenatal exposure to maternal cells and tissues in priming NIMA-specific tolerance.

Nonetheless, this apparent requirement for postnatal ingestion of maternal antigens through breastfeeding opens up an instructive experimental window for probing how NIMAspecific tolerance is sustained in offspring. In turn, the potent immunomodulatory effects of maternal cells in breast milk suggest that it may be possible to therapeutically optimize their beneficial properties in offspring. As pointed out by Moles and colleagues in their Correspondence ${ }^{11}$, breast milk and colostrum contain different immune cell populations, including memory lymphocytes, professional antigen-presenting cells, along with embryonic and mesenchymal stem cells. Given this diversity, further dissecting the unique immunological and non-immunological benefits of each cell subset will shed important new light on how microchimeric cells influence health and disease.
Jeremy M. Kinder, Ina A. Stelzer and Sing Sing Way are at the Division of Infectious Disease and Perinatal Institute at Cincinnati Children's Hospital, 3333 Burnet Avenue, MLC 7017, Cincinnati, Ohio 45229 USA.

Petra C. Arck is at the Laboratory of Experimental Feto-Maternal Medicine, Department of Obstetrics and Prenatal Medicine, University Medical Center Hamburg-Eppendorf, 20246 Hamburg, Germany.

Correspondence to S.S.W. singsing.way@cchmc.org doi: $10.1038 /$ nri. 2017.117 Published online 3 Oct 2017

1. Kinder, J. M. et al. Immunological implications of pregnancy-induced microchimerism

Nat. Rev. Immunol. 17, 483-494 (2017).

2. Dutta, P. \& Burlingham, W. J. Tolerance to noninherited maternal antigens in mice and humans. Curr. Opin. Organ Transplant. 14, 439-447 (2009).

3. Nelson, J. L. The otherness of self: microchimerism in health and disease. Trends Immunol. 33, 421-427 (2012).

4. Kinder, J. M. et al. Cross-generational reproductive fitness enforced by microchimeric maternal cells. Cell 162, 505-515 (2015).

5. Owen, R. D. et al. Evidence for actively acquired tolerance to Rh antigens. Proc. Natl Acad. Sci. USA 40, 420-424 (1954).

6. Khosrotehrani, K. et al. Presence of chimeric maternally derived keratinocytes in cutaneous inflammatory diseases of children: the example of pityriasis lichenoides. J. Invest. Dermatol. 126 345-348 (2006)

7. Nelson, J. L. et al. Maternal microchimerism in peripheral blood in type 1 diabetes and pancreatic islet $\beta$ cell microchimerism. Proc. Natl Acad. SCi. USA 104, 1637-1642 (2007).

8. Stevens, A. M. et al. Myocardial-tissue-specific phenotype of maternal microchimerism in neonatal lupus congenital heart block. Lancet 362 , 1617-1623 (2003).

9. Ye, J., Vives-Pi, M. ¿ Gillespie, K. M. Maternal microchimerism: increased in the insulin positive compartment of type 1 diabetes pancreas but not in infiltrating immune cells or replicating islet cells. PLOS ONE 9, e86985 (2014).

10. Harrington, W. E. et al. Maternal microchimerism predicts increased infection but decreased disease due to Plasmodium falciparum during early childhood. J. Infect. Dis. 215, 1445-1451 (2017).

11. Moles, J. P. et al. Breastfeeding related maternal microchimerism. Nat. Rev. Immunol. http://dx.doi. org/10.1038/nri .2017.115 (2017)

12. Burlingham, W. J. et al. The effect of tolerance to noninherited maternal HLA antigens on the survival of renal transplants from sibling donors. N. Engl. J. Med. 339, 1657-1664 (1998)

13. Campbell, D. A. Jr et al. Breast feeding and maternaldonor renal allografts. Possibly the original donor-specific transfusion. Transplantation 37, 340-344 (1984).

14. Andrassy, J. et al. Tolerance to noninherited maternal $\mathrm{MHC}$ antigens in mice. J. Immunol. 171, 5554-5561 (2003).

15. Mold, J. E. et al. Maternal alloantigens promote the development of tolerogenic fetal regulatory T cells in utero. Science 322, 1562-1565 (2008).

\section{Acknowledgements}

The writing of this correspondence was made possible through funding by Cusanuswerk-Studienförderung (to I.A.S.); Deutsche Forschungsgemeinschaft (AR232/25-1 in KFO296 and AR232/27-1 to P.C.A.); the US National Institutes of Health, Office of the Director (DP1AI 131080 to S.S.W.); the US National Institute of Allergy and Infectious Disease (RO1AI100934 and R01Al120202 to S.S.W.); and the March of Dimes Foundation (FY15-254 to S.S.W.). S.S.W. is a Burroughs Wellcome Fund Investigator in the pathogenesis of infectious disease and Howard Hughes Medical Institute Faculty Scholar.

\section{Author contributions}

All authors contributed to researching and reviewing the data for this correspondence. S.S.W. drafted this correspondence with editorial input from all the authors.

Competing interests statement

The authors declare no competing interests. 